\title{
Nonunion of fibula: a systematic review
}

\author{
Arup K. Bhadra • Craig S. Roberts • \\ Peter V. Giannoudis
}

Received: 6 March 2012 / Accepted: 11 April 2012 / Published online: 30 May 2012

(C) Springer-Verlag 2012

\begin{abstract}
Purpose The purpose of this systematic review was to analyse the available evidence regarding nonunions of the fibula. We focussed on the incidence, risk factors, evaluation, and treatment modalities for fibular nonunions as evident in the current literature and propose a treatment algorithm.

Methods This was an Institutional Review Board (IRB) exempt study performed at a level one trauma centre. We systematically reviewed the published evidence on fibular nonunion or delayed union from 1950 to February, 2011.

Results Twelve articles were included in this systematic review. In summary, nonunion of the fibula is becoming increasingly more common in association with intramedullary nailing of concomitant tibial shaft fractures. A treatment algorithm for nonunion of the fibula has been proposed.

Conclusions The suspicion for nonunion of the fibula should be heightened in lower leg fractures if the patient is symptomatic, and the progression of healing is not as expected. Ideally, prospective, multicentre studies would be performed to provide more rigorous data on the incidence, risk factors, and optimum treatment.
\end{abstract}

\footnotetext{
A. K. Bhadra • C. S. Roberts $(\bowtie)$

Department of Orthopaedic Surgery, University of Louisville, 550 S. Jackson Street, 1st Floor, ACB, Louisville, KY 40202, USA

e-mail: craig.roberts@louisville.edu

P. V. Giannoudis

Department of Orthopaedic Surgery, University of Leeds, Beckett Street,

Leeds LS9 7TF, UK
}

\section{Introduction}

Fibular fracture may occur in conjunction with fractures of the tibia or as a component of malleolar fracture of the ankle. The true incidence of the nonunion of the fibula (lateral malleolar fracture in conjunction with ankle injury and fibula fracture in conjunction with a tibial shaft fracture) is difficult to determine because most remain asymptomatic and, therefore, unrecorded.

The fibula is fractured in $75-85 \%$ of cases with fractures of the tibia [1]. Most of these fractures heal without consequences; some do not heal and go on to become nonunions. Although recent evidence suggests that the incidence of nonunion of the fibula has risen with the interlocking intramedullary nailing of the tibia, there is a scarcity of information on delayed union or nonunion of fibula in conjunction with a concomitant tibial fracture.

Although the nonunion of the medial malleolus has been a subject of a fair number of articles, ununited fractures of the lateral malleolus (fibular nonunions) have been generally regarded as a complication of little or no significance [2].

The purpose of this systematic review was to analyse the available evidence regarding nonunions of the fibula. We focussed on the incidence, risk factors, evaluation and treatment modalities for fibular nonunions as evident in the current literature and have proposed a treatment algorithm.

\section{Materials and methods}

This was an Institutional Review Board (IRB) exempt study performed at a level one trauma centre. We performed a PubMed search using the following MeSH words: 'fibula fracture' $[\mathrm{MeSH}]$ ununited fibula' [MeSH], 'fibula + nonunion' [MeSH], 'pseudoarthrosis lateral malleolus' [MeSH]. 
An Ovid Medline search was also conducted, and a review of the references listed in all articles to be included in the metaanalysis in an attempt to find more potentially relevant articles.

Inclusion and exclusion criteria

We systematically reviewed the published evidence on fibular nonunion or delayed union from 1950 to February, 2011. Studies included in this review needed to have populations of adults who sustained fibular fractures as a part of an ankle injury or in conjunction with tibia fractures. The articles or the abstracts needed to be in the English language, reporting two or more cases of delayed union, nonunion of the fibula and/or pseudoarthrosis of the lateral malleolus. Single case reports or cases involving the paediatric population were excluded. Case series, prospective cohort, and retrospective reviews were included. Review articles without patient data were excluded.

\section{Level of evidence}

All the studies were retrospective case series (evidencebased medicine - level IV).

\section{Results}

The PubMed MeSH search yielded 236 results (Fig. 1). Although the Ovid Medline search yielded 7,948 results, it did not show any additional studies meeting our eligibility criteria. The reference review of all articles contributed four additional studies complying with our eligibility criteria. Seventy-six articles were excluded because the index procedures and cause of the nonunion was a fibular strut graft used for another nonunion site. Sixty articles described nonunion of other bones. Thirty-eight articles were on unrelated topics on basic science research. There were 21 articles on biomechanical studies. Eighteen other articles were excluded because 12 were determined to be unrelated case reports (e.g., paediatric fractures, paediatric pseudoarthrosis, etc.) and six were on ankle/fibular malunion. Twenty-three articles describing fibular nonunion/delayed union or pseudoarthrosis were reviewed. Twelve articles were included in this systematic review. Six articles and five single case reports which dealt with the paediatric population were excluded.

Incidence

There is very little evidence on true incidence of isolated fibular nonunion. It has been reported to occur either as a part of ankle fracture or in combination with fractures of the tibia (Fig. 2-5). The overall incidence varies from $0.3 \%$ to $5.4 \%$. A total of 119 fibular nonunion cases were reported, of which 69 (57.9\%) were part of a rotational ankle fracture, 49 (41.2\%) accompanied fracture of the tibial shaft and one $(0.8 \%)$ nonunion followed

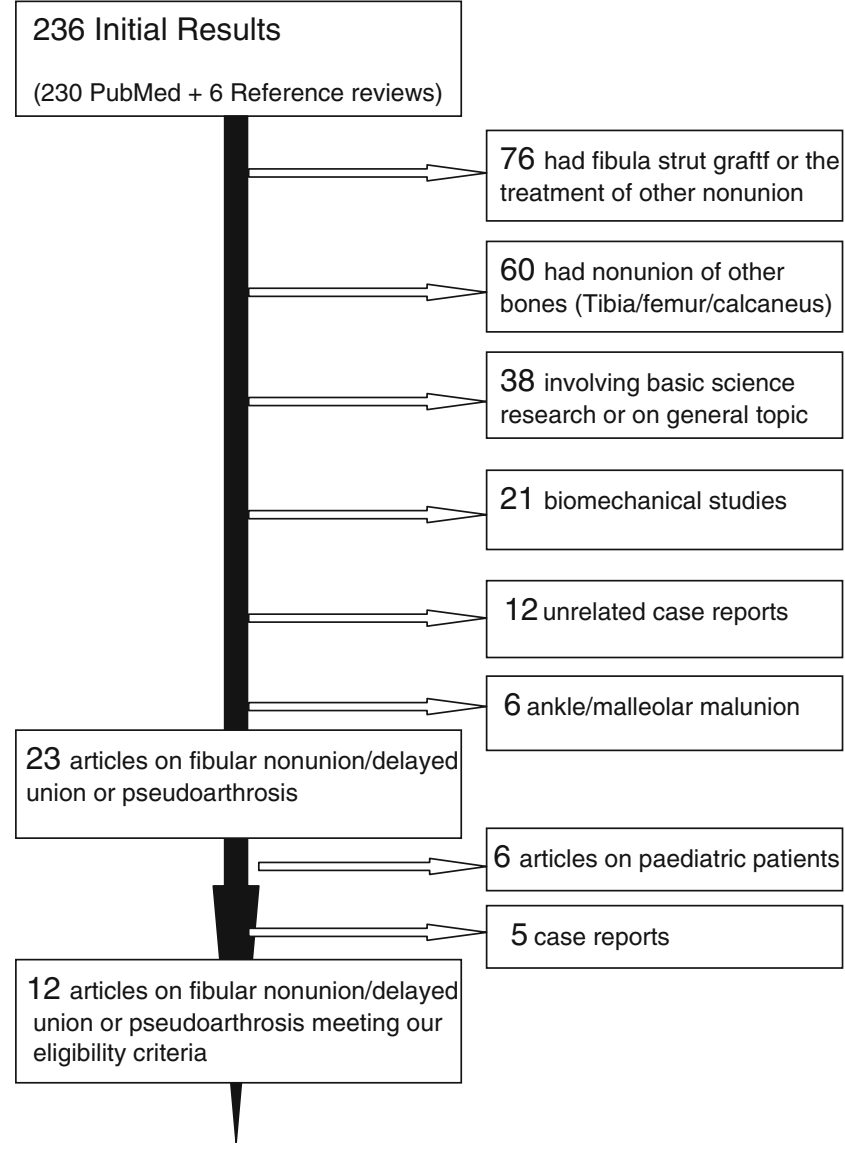

Fig. 1 Flow chart depicting articles exclusion breakdown

fibular osteotomy (Table 1). In the five articles which reported the gender of patients $[1,3-6]$, the male to female ratio was 2.9:1. The age range was from ten years to 72 years. The range of follow-up (from three months to 23 years) was reported in eight articles only [1-4, 7-10]. In combined tibia/fibula fractures, fibular nonunion occurred more commonly when the tibia was stabilised with intramedullary nails $(5.5 \%$ in the nail group, $4.14 \%$ in the plate group, and none in the conservative group) $[1,4,5]$. Ankle fractures involving the medial malleolus showed a higher incidence of lateral malleolar pseudoarthrosis $(0.7 \%)$ than fractures which did not involve the medial side (0.1\%) [3].

Aetiology and risk factors

A poor biological or mechanical environment at the fracture site or a combination of the two plays a significant role leading to nonunion [11]. Inadequate blood supply can be caused by trauma, surgical disruption, or by instability at the fracture site. Instability is the principal mechanical factor that leads to aseptic nonunions. Ebraheim et al. [4] described fibular nonunion as a potential indicator of instability of the fixation at the tibial fracture site as the authors documented 16/17 (94\%) fibular nonunions to be associated with nonunion or delayed union of the tibia. The other important factors associated with delayed or nonunion of 
Fig. 2 Anteroposterior (AP) and lateral radiographs of tibia and fibula with tibial intramedullary nail in situ, showing hypertrophic nonunion of fibula (white arrow)

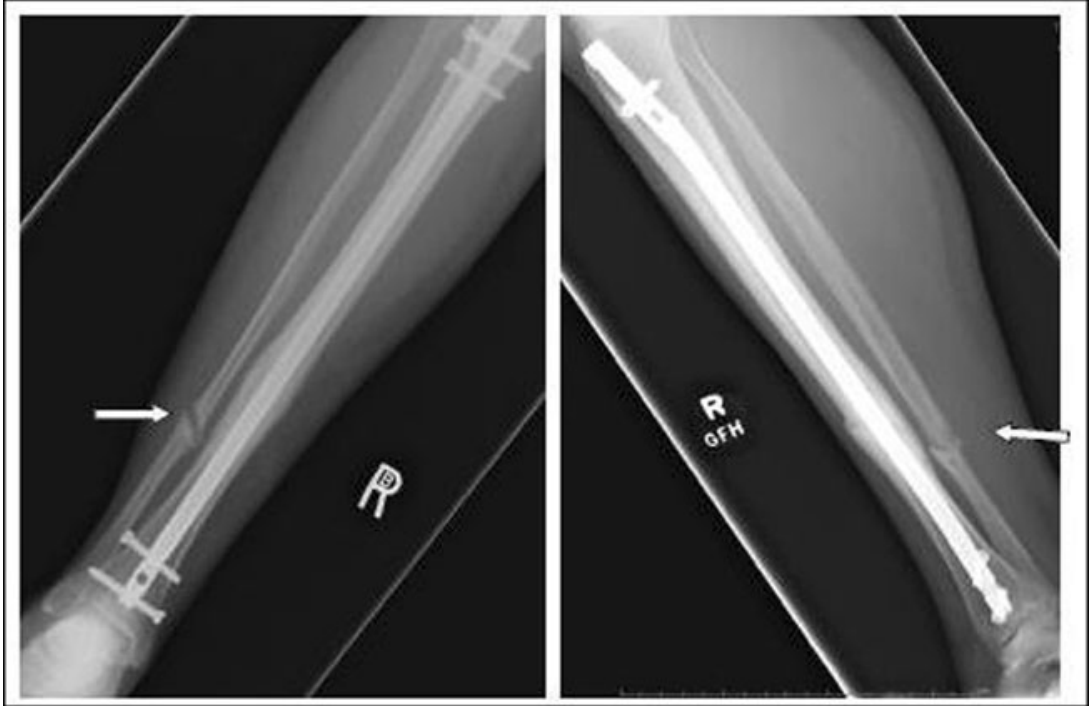

the fibula were fracture displacement, oblique fracture pattern, initial fracture comminution, high-energy injuries, and initial treatment by internal fixation $[1,12,13]$. There was no consensus about the association of nonunion with open fracture. Böstman and Kyrö [1] reported 3/8 (37.5\%) of fibular nonunion being associated with open tibial fracture, but there was no skin lesion over the fibular fracture. Shen and Shen [5] and Siliski et al. [13] suggested no significant association of open wound with subsequent nonunion. If there is malrotation of the distal fibula, especially in cases of rotational low-energy ankle fractures, the trabecular linearity changes, leading to undue stress at fracture site which could be a contributing factor to nonunion [8].

\section{Evaluation and diagnosis}

\section{Clinical evaluation}

In over $80 \%$ of patients (Table 2 ), the most common presentation was pain over the lateral aspect of the ankle joint or over the fibular fracture site which was worse on exertion. Patients also presented with fracture site tenderness, ankle swelling,
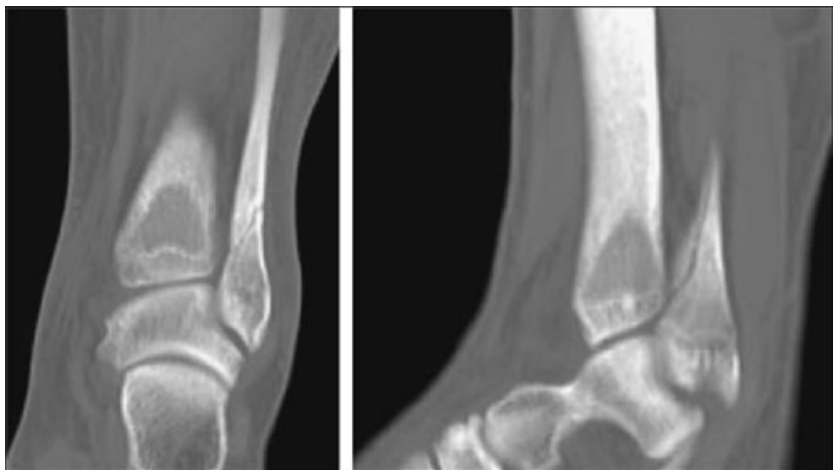

Fig. 3 CT scans showing atrophic non-union of fibula paraesthesia $[1,2,5]$, sometimes instability, and often with an antalgic gait [3]. In less than $20 \%$ of patients, the fracture was completely asymptomatic, and nonunion of the fibula was an incidental radiological finding $[1,3]$. There was no consensus regarding the time from injury to diagnosis of delayed union or nonunion. Diagnosis ranged from five weeks to 52 weeks, but was commonly 24 weeks or beyond (Table 1 ).

\section{Radiological evaluation}

All patients were diagnosed or initially suspected of having delayed union or nonunion of the fibula on plain
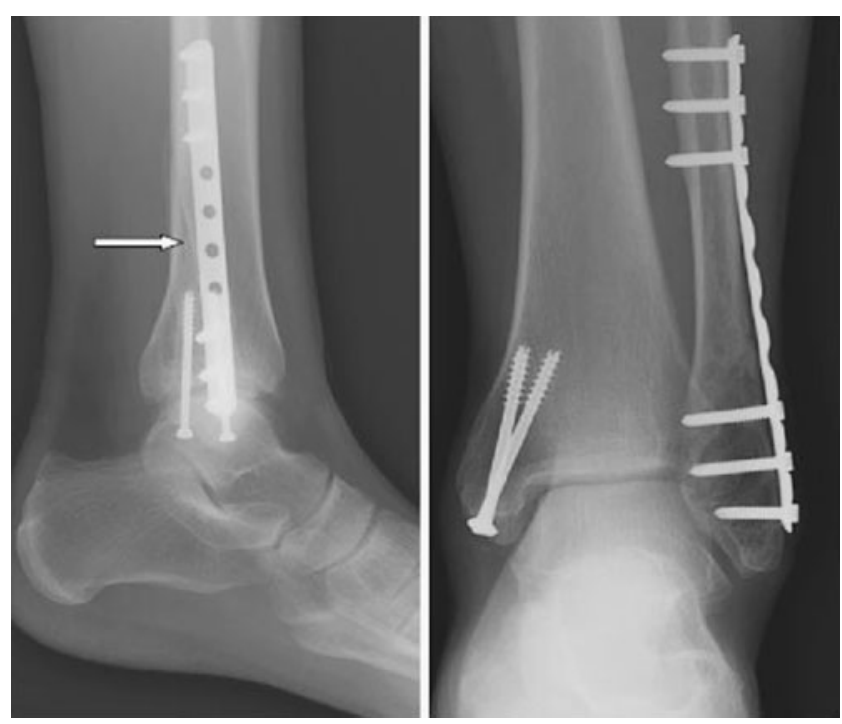

Fig. 4 Lateral and anteroposterior (AP) views of ankle with internally fixed Weber B type fibula fracture five months post-surgery. The fracture line is barely visible on lateral X-ray (white arrow), but difficult to appreciate in AP view. The patient had a CT scan (Figure 4) confirming atrophic nonunion 
Fig. 5 Axial, sagittal and coronal views of CT scan of ankle showing incomplete nonunion (white arrow) of internally fixed Weber B type fibula fracture
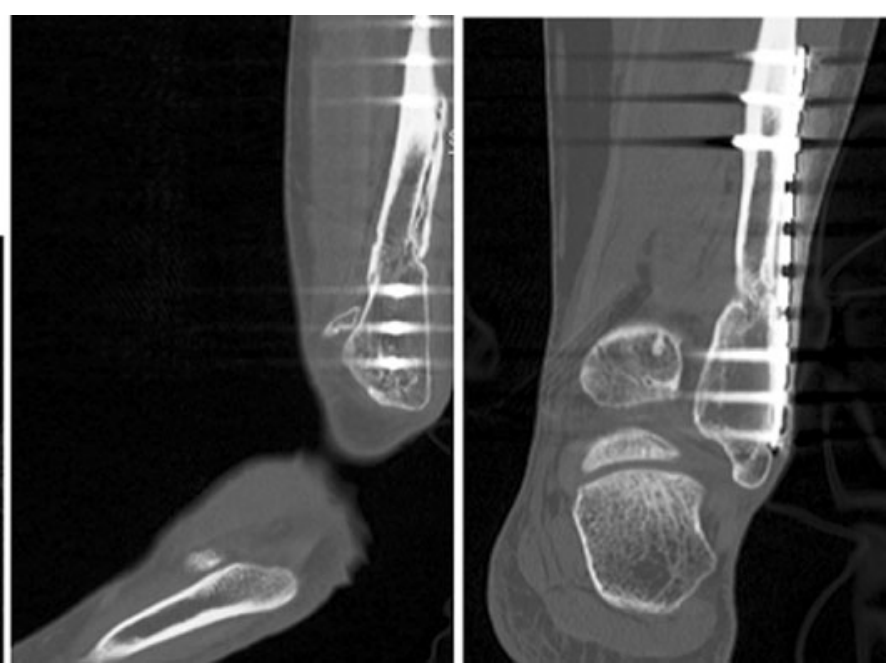

X-rays. CT scan as a diagnostic radiological investigation was used in only less than $20 \%$ of the cases. CT scan was more useful in cases with suspected concomitant incomplete union of the tibia [4] and in cases of Weber B type ankle fractures [6, 8-10] where the extent of fibular union was sometimes difficult to appreciate on plain X-rays (Fig. 4). Böhler [14] stated that a case could not be called pseudoarthrosis unless a gap of more than $3 \mathrm{~mm}$ between the fracture surfaces remained six months after the fracture. Mendelsohn [2] used the concept of nonunion in connection with malleolar fracture when a fracture line at least two to three millimetres wide and sclerosing of the fracture surfaces were present at least six months after the fracture.

\section{Classification}

Traditionally, a nonunion is classified as either hypertrophic or atrophic depending on the pattern of callus formation on the radiograph. Hypertrophic nonunions of the fibula (Fig. 1) were found to be more symptomatic and painful $[1,5,12]$. In contrast, the majority of atrophic nonunions (Fig. 2) were asymptomatic $[1,5]$. Nonunion of the fibula was also classified as complete or incomplete. In complete nonunion, there was no sign of any bridging callus visible in radiographs, and this pattern was more commonly seen in Weber A and C type fibular fractures. When there was partial union of fracture surfaces, it was called incomplete nonunion, and this pattern was mostly seen in Weber B type injuries [6].

Table 1 Overview of selected articles

\begin{tabular}{|c|c|c|c|c|c|c|c|}
\hline Authors & $\begin{array}{l}\text { Number } \\
\text { of fibular } \\
\text { nonunions }\end{array}$ & $\begin{array}{l}\text { Total patients } \\
\text { treated for } \\
\text { ankle or tibia/ } \\
\text { fibular fractures }\end{array}$ & Incidence & Age in years & $\begin{array}{l}\text { Male: } \\
\text { Female }\end{array}$ & $\begin{array}{l}\text { Time to } \\
\text { diagnosis } \\
\text { (weeks) }\end{array}$ & Site of fibula fracture \\
\hline Mendelsohn [2] & 2 & 190 & $1.1 \%$ & - & - & - & Distal 1/3 (2) \\
\hline Sneppen [3] & 23 & 4760 & $0.3 \%$ & $20-68$ & $21: 2$ & $>36$ & Malleolar or distal 1/3 (23) \\
\hline Sneppen [7] & 6 & - & - & $10-62$ & - & $>24$ & Distal 1/3 (6) \\
\hline Konig and Gotzen [12] & 6 & - & - & - & - & - & Distal 1/2 (6) \\
\hline Böstman and Kyrö [1] & 8 & 440 & $5.4 \%$ & $21-54$ & $6: 2$ & $>16$ & Distal 1/3 (2) Middle 1/3 (6) \\
\hline Shen and Shen [5] & 15 & 335 & $4.5 \%$ & $17-72$ & $3.5: 1$ & - & - \\
\hline Ebraheim et al. [4] & 17 & - & - & $18-63$ & $15: 2$ & $>24$ & $\begin{array}{l}\text { Distal 1/3 (11) Middle 1/3 (3) } \\
\text { Proximal 1/3 (1)Segmental (2) }\end{array}$ \\
\hline Siliski et al. [13] & 26 & - & - & - & - & - & Distal 1/3 (26) \\
\hline Walsh and DiGiovanni [6] & 6 & 17 & - & $42-63$ & $1: 5$ & $5-52$ & Distal 1/3 (6) \\
\hline Ahmed et al. [8] & 3 & - & - & $37-42$ & $1: 2$ & 18 & Distal 1/3 (3) \\
\hline Brinker and O'Connor [9] & 4 & - & - & $18-70$ & $2: 2$ & - & Distal 1/3 (2) Middle 1/3 (2) \\
\hline McGonagle et al. [10] & 3 & - & - & $21-52$ & $3: 0$ & $14-56$ & Distal 1/3 (3) \\
\hline Total $=12$ & 119 & 5742 & $0.3 \%-5.4 \%$ & $10-72$ & $2.9: 1$ & & $\begin{array}{c}\text { Distal 1/3 }(90 ; 86.5 \%) \\
\text { Mid } 1 / 3(11 ; 10.5 \%) \\
\text { Proximal } 1 / 3(1 ; 0.9 \%)\end{array}$ \\
\hline
\end{tabular}


Table 2 Associated injuries or fracture pattern

\begin{tabular}{|c|c|c|c|c|c|c|}
\hline \multirow[t]{2}{*}{ Authors } & \multirow[t]{2}{*}{ Patients } & \multirow[t]{2}{*}{ Associated injury or type of injury } & \multicolumn{2}{|l|}{ Symptoms } & \multicolumn{2}{|l|}{ Diagnosis } \\
\hline & & & None & $\begin{array}{l}\text { Pain/tenderness } \\
\text { paraesthesia }\end{array}$ & $\mathrm{X}$-ray & $\mathrm{CT}$ \\
\hline Mendelsohn [2] & 2 & Bimalleolar fracture (1) & - & 2 & 2 & - \\
\hline Sneppen [3] & 23 & $\begin{array}{l}\text { Bimalleolar fracture (9) Trimalleolar } \\
\text { fracture (4), SA (16), SE (6), PA (1) }\end{array}$ & 6 & 17 & 23 & - \\
\hline Sneppen [7] & 6 & Bimalleolar fracture (1) & - & 6 & 6 & - \\
\hline Konig and Gotzen [12] & 6 & Distal tibia fracture (6) & - & 6 & 6 & - \\
\hline Böstman and Kyrö [1] & 8 & Middle 1/3 tibia fracture (8) & 3 & 5 & 8 & - \\
\hline Shen and Shen [5] & 15 & Distal tibia fracture (15) & 11 & 4 & 15 & - \\
\hline Ebraheim et al. [4] & 17 & $\begin{array}{l}\text { Distal 1/3 tibia fracture (11) Middle 1/3 tibia } \\
\text { fracture (3) Segmental tibia fracture (3) }\end{array}$ & 1 & 16 & 17 & 4 \\
\hline Siliski et al. [13] & 26 & $\begin{array}{l}\text { Weber A type (2) Weber B type (4) } \\
\text { Weber C type (20) }\end{array}$ & - & 26 & - & - \\
\hline Walsh and DiGiovanni [6] & 6 & $\begin{array}{l}\text { Weber A type (1) Weber B type (4) } \\
\text { Weber C type (1) }\end{array}$ & - & 6 & 6 & 4 \\
\hline Ahmed et al. [8] & 3 & Weber B 3 & - & 3 & 3 & 3 \\
\hline Brinker and O'Connor [9] & 4 & $\begin{array}{l}\text { Distal 1/3 tibia fracture (2) Middle 1/3 } \\
\text { tibia fracture (1) Fibular osteotomy (1) }\end{array}$ & - & 4 & 4 & 4 \\
\hline McGonagle et al. [10] & 3 & Distal 1/3- 3 & 1 & 2 & 3 & 3 \\
\hline Total & 119 & $\begin{array}{l}\text { Ankle fracture }(69 ; 57.9 \%) \text { Tib/Fib fracture } \\
(49 ; 41.2 \%) \text { Fibular osteotomy }(1 ; 0.8 \%)\end{array}$ & $22(18.5 \%)$ & $97(81.5 \%)$ & $93(100 \%)$ & $18(19.3 \%)$ \\
\hline
\end{tabular}

$S A$ supination adduction, $S E$ supination external rotation, $P A$ pronation abduction

\section{Fracture site}

The most common site $(86.5 \%)$ of fracture nonunion involved fractures of the distal third of the fibula (Table 1). Less soft tissue coverage, and thus, blood supply, increased micromotion [15]. Therefore, relative instability was postulated as a possible explanation behind the higher risk of nonunion at the distal end of the fibula. The nonunion rate was approximately $10 \%$ at the level of the middle third of the fibula and rare $(<1 \%)$ at the proximal end. This was probably due to better muscle and soft tissue envelopes and the close proximity of the nutrient artery to the proximal end of the fibula [6].

\section{Associated injuries or fracture pattern}

Fibular fracture occurs either as a part of rotational ankle fractures or in conjunction with fractures of the tibia. Of the reported nonunions of the fibula, $57.9 \%$ were in the ankle fracture group and the remaining $41.2 \%$ were in combination with a tibial shaft fracture (Table 2). Seventy percent $(34 / 48)$ of the cases of nonunion of the fibula in combined fractures of tibia-fibula was observed when the distal third was involved, but it was only $25 \%$ (12/48) when accompanying fractures involved the middle third of the tibia. Weber C type fractures were more prone to nonunion [13]. Sneppen [3] described medial instability was an important factor in the aetiology of lateral malleolus pseudoarthrosis. A higher incidence of lateral malleolar pseudoarthrosis was noted in bimalleolar or trimalleolar ankle fractures. The author also noticed that ankle fractures of a supination type (supination adduction) seemed to develop lateral malleolar pseudoarthosis in a majority (69\%) of cases. Walsh and DiGiovanni [6] demonstrated the highest incidence (66\%) of fibular nonunion in Weber B type fractures; but interestingly, most of them were partial nonunions.

\section{Treatment}

There were a variety of treatment modalities documented in the literature for nonunion of the fibula (Table 3). Treatment is based on the type of nonunion, symptomatology, initial fracture pattern, associated injuries and patient's wishes.

\section{Conservative management}

Observation Although only less than $20 \%$ patients with fibular nonunion were asymptomatic, nonoperative treatment was the most commonly practiced mode of treatment (44.6 \%). Böstman and Kyrö [1] found that $50 \%$ of nonunion of fibular fractures accompanying tibial shaft fractures went on to eventual bony union without any intervention. The authors concluded that delayed fibular union was a prolonged repair process continuing for several years after the initial injury resulting ultimately in spontaneous union. Shen and Shen [5] treated $87 \%(13 / 15)$ of nonunion of the 
Table 3 Treatment

\begin{tabular}{|c|c|c|c|c|c|c|c|c|c|}
\hline \multirow[t]{2}{*}{ Authors } & \multirow[t]{2}{*}{ Patients } & \multicolumn{8}{|l|}{ Treatment } \\
\hline & & Obs & $\begin{array}{l}\text { External } \\
\text { fixator }\end{array}$ & $\begin{array}{l}\text { ORIF } \\
\pm \mathrm{BG}\end{array}$ & BG only & Arthrodesis & Excision & Drilling & $\begin{array}{l}\text { Segmental } \\
\text { resection }\end{array}$ \\
\hline Mendelsohn [2] & 2 & - & - & - & 1 & 1 & - & - & - \\
\hline Sneppen [3] & 23 & 20 & - & 3 & - & - & - & - & - \\
\hline Sneppen [7] & 6 & - & - & 2 & 2 & - & 1 & 1 & - \\
\hline Konig and Gotzen [12] & 6 & 2 & - & 4 & - & - & - & - & - \\
\hline Böstman and Kyrö [1] & 8 & 7 & - & - & - & - & - & - & 1 \\
\hline Shen and Shen [5] & 15 & 13 & - & - & - & - & - & - & 2 \\
\hline Ebraheim et al. [4] & 17 & 2 & 4 & 8 & 3 & - & - & - & - \\
\hline Siliski et al. [13] & 26 & 7 & - & 18 & - & 1 & - & - & - \\
\hline Walsh and DiGiovanni [6] & 6 & - & - & 4 & 1 & - & 1 & - & - \\
\hline Ahmed et al. 2007 & 3 & - & - & 3 & - & - & - & - & - \\
\hline Brinker and O'Connor [8] & 4 & - & - & - & - & - & - & - & 4 \\
\hline McGonagle et al. [10] & 3 & 1 & - & 2 & - & - & - & - & - \\
\hline Total & $119(100 \%)$ & $52(43.6 \%)$ & $4(3.4 \%)$ & $44(36.9 \%)$ & $7(5.9 \%)$ & $2(1.6 \%)$ & $2(1.6 \%)$ & $1(0.8 \%)$ & $7(5.9 \%)$ \\
\hline
\end{tabular}

Obs observation, $O R I F$ open reduction and internal fixation, $B G$ bone grafting

fibula fractures combined with fractures of tibia shaft conservatively and suggested that primary fixation of the fibula was not needed. Sneppen [3] described that lateral malleolus pseudoarthrosis as a part of ankle fractures showed a marked tendency to spontaneous union.

Electrical stimulation Electrical and magnetic devices were created to treat nonunions based on the fact that electric fields occur in mechanically loaded bones. Three types of electrical stimulation are available for healing of fracture or nonunions: (1) direct current stimulation using electrodes, (2) electromagnetic stimulation, and (3) capacitive coupling stimulation [16]. Cells from atrophic nonunion should respond slower to electromagnetic field stimulation than the cells from hypertrophic nonunion [17]. Siliski et al. [13] reported the successful healing of fibular nonunion in 18 cases in which nonunion was treated with open reduction and internal fixation, with or without bone grafting or electrical stimulation. The authors, however, did not specify the exact number of cases treated with electrical stimulation in this series.

External fixation Four cases (3.4\%) were treated with external fixation. There was also an underlying nonunion/delayed union of a concomitant fracture of the tibia. Three cases $(75 \%)$ eventually healed at 12 months, and one (25\%) still remained united at six months [4].

Drilling Drilling of pseudoarthrosis is performed to stimulate osteosynthesis. Sneppen [7] treated one case (16\%) of lateral malleolar pseudoarthrosis with drilling and reported bony union at around four months.
Surgical The indications for surgery included persistent, reproducible pain, fracture site tenderness, antalgic gait, instability, and radiographic and/or CT evidence of nonunion [1-25].

Open reduction internal fixation (ORIF) \pm bone grafting (BG) Open reduction and internal fixation in direct compression mode with or without bone grafting results in successful healing of symptomatic fibular nonunions. Forty-four cases $(36.9 \%)$ of all nonunions were treated with $\mathrm{ORIF} \pm \mathrm{BG}$. Hypertrophic nonunion primarily requires fracture site stabilization with compression plating. Bone grafting was not routinely needed unless there was significant malalignment or bone defect [23]. Atrophic nonunion required both fracture stability and optimum biological environment at the fracture site (Fig. 3). Often, it was necessary to resect the nonviable bone and fibrous tissue, and the defect was filled with autologus bone graft [7]. Konig and Gotzen [12] reported complete alleviation of symptoms and bony union in four cases $(66 \%)$ which were treated with plate osteosynthesis of the fibula. Ebraheim et al. [4] treated eight cases (47\%), Walsh and DiGiovanni [6] treated four cases (66\%) and Siliski et al. [13] treated 18 cases (69\%) with plate osteosynthesis and autologus bone grafting and reported good outcome with bony union in all cases. In recent years, the majority of symptomatic Weber B fracture nonunions have been successfully treated with ORIF and autologus bone grafting $[6,8,10]$.

Bone graft only Seven cases (5.9\%) of nonunion of the fibula were treated with bone grating of the fracture site only. Sneppen [7] treated two cases of lateral malleolar pseudoarthosis with an autologous inlay bone graft applied on the pseudoarthrosis defect without resecting the fibrous tissue, and healing occurred 
within three months in one case and within a year in the other. Mendelsohn treated one case with autologous bone graft only, but the patient remained symptomatic even after 18 months [2]. Walsh and DiGiovanni reported significant symptomatic improvement in a case of nonunion of the fibula treated with burr resection and curettage of the nonunion with autologous bone grafting harvested from the calcaneus [6]. Ebraheim et al. [4] treated three cases of nonunion of the fibula with an accompanying tibial fracture with posterolateral bone graft only and reported bony union in two cases between 19 and 28 months. The third patient was lost in follow-up.

Arthrodesis Two fibular nonunion cases (1.6\%) required ankle arthrodesis [2, 13]. One of them, an open ankle fracture, was treated with immediate internal fixation and subsequently developed a chronic infection.

Excision If the ununited fragment of the fibula was very small and distal, it was excised in the symptomatic patients. Two cases $(1.6 \%)$ were treated with excision $[6,7]$ of the ununited distal piece; one of them had lateral ligament (modified Bröstrom) reconstruction to provide continued ankle stability postoperatively [6].

Segmental resection Segmental resection or partial fibulectomy provided significant symptomatic relief in a patient with painful hypertrophic fibular nonunion. One to five centimetres of the fibula around the nonunion site was resected. Generally, it should be considered in nonunions involving the middle third of the shaft of the fibula. In order to preserve normal ankle and knee mechanics, the published recommendation is to make the most distal and proximal resection a minimum of five to six centimetres from the respective ends of the fibula $[9,24]$. Overall, seven cases $(5.9 \%)$ were treated by segmental resection which resulted in complete resolution of pain $[1$, $5,9]$.

\section{Discussion}

Our systematic review of the orthopaedic literature noted that less than adequate attention has been paid to fibular nonunion. Fibular fracture may occur as a component of lower energy rotational ankle injury or in conjunction with high-energy tibial fractures. Although most of these fractures heal without consequences, a few go on to cause problems. Among the long bones, the fibula undoubtedly possesses ideal features for successful fracture healing. The soft tissue coverage is ample and the endosteal vascular supply is good, too [20]. Moreover, the fibula is subjected to small mechanical stresses compared to the tibia. Nonunion of fibula is an entity which is rare, but exists. With the advent of intramedullary nailing of the concomitant tibial shaft fracture, a trend of higher fibular nonunion is noticed.

Many authors have tried to define nonunion or pseudoarthrosis over the last half century $[2,3,14,18]$. Nonunion of the fracture is defined as "cessation of all reparative process of healing without bone union" [11]. In the absence of significant bone loss, nonunion is usually diagnosed between six and nine months following the fracture. The term pseudoarthosis was originally applied to that form of absent bony healing in which the ends of the fragments are sclerotic covered by cartilage and shut off from the medullary cavity as by a lid and by a joint capsule. In German and English, the terms pseudoarthrosis and nonunion are synonymous [22, 25]. According to AO Principles of Fracture Management, delayed union describes the situation where there are distinct clinical and radiological signs of prolonged fracture healing. The difference between the terms delayed and nonunion is one of time [25].

Twelve articles met our search criteria and were the focus of this systematic review. All of the studies were retrospective case series (evidence-based medicine [EBM] - level IV). We focused on the incidence, clinical evaluation, risk factors, associated injuries and management of delayed or nonunion involving the fibula. The true incidence of fibular nonunion is difficult to determine as many of them do not constitute any major clinical problem. Our overall finding suggests a higher incidence of delayed or nonunion of the fibula in association with concomitant shaft fractures of the tibia (4.5-5.4\%) to that with rotational ankle fractures $(0.3-1.1 \%)$. Nonunion was most commonly ( $>80 \%)$ seen in fractures involving the distal third of the fibula. Fibular nonunion in rotational ankle fracture was higher in Weber type B fractures.

Although approximately $20 \%$ of cases with fibular nonunion may be asymptomatic, persistent pain over the fracture site was the most common presentation $(81.5 \%)$. Paresthesia, fracture site, tenderness, and instability of the ankle were the other modes of presentation. We agree with the statement from Walsh and DiGiovanni, “... clinicians need to have a heightened suspicion of potential occult fibular nonunion in patients with stable rotational ankle fracture who fail to progress as expected" [6].

The exact cause of fibular nonunion mostly remained unclear in our study. Although the parameters of age, gender, and open versus closed fracture were not found to have prognostic significance with regard to fibular nonunion $[5,12,13]$, initial fracture pattern, severity of communition at the fracture site, degree of displacement of fracture fragments, and high-energy injury seemed to be related to the occurrence of delayed fibular union or frank nonunion $[1,12,13]$. The distal third of the fibula was the most common site ( $86.5 \%$ ) for nonunion or pseudoarthrosis in both combined tibia-fibula fractures and low-energy ankle fractures. The distal fibula, although it only bears a small fraction of the weight-bearing surface of the joint through the syndesmosis, is subject to torsion, shear, rotation 
and compression. It is this inherent subjection to such forces that make it susceptible to nonunion [6].

Nonunion of the fibula may indicate instability of the concomitant fracture of tibia [4]. It may be necessary to enhance the stability of the tibia in order to facilitate fibular union. Although the majority of fibular nonunion can be diagnosed with plain radiographs, CT scan is useful particularly for partial nonunion of the fibula in Weber type B ankle fractures and occult instability of the tibia in combined tibia-fibula fractures.
Due to their benign nature, many of the fibular nonunions $(43.6 \%)$ were managed using nonoperative treatment; and spontaneous union ultimately occurred, in many cases, after a prolonged period from the injury. For symptomatic nonunion of the fibula, plate fixation with or without autologus bone grafting (36.9\%) resulted in complete resolution of symptoms and eventual bony union $[6-8,10,12]$. Although there are other modalities like percutaneous autologus bone marrow grafting [21], osteoinductive molecules like BMP, and TGF$\beta 1$ [19] which have been used to treat nonunions of long
Fig. 6 Flow chart depicting treatment algorithm for nonunion of fibula

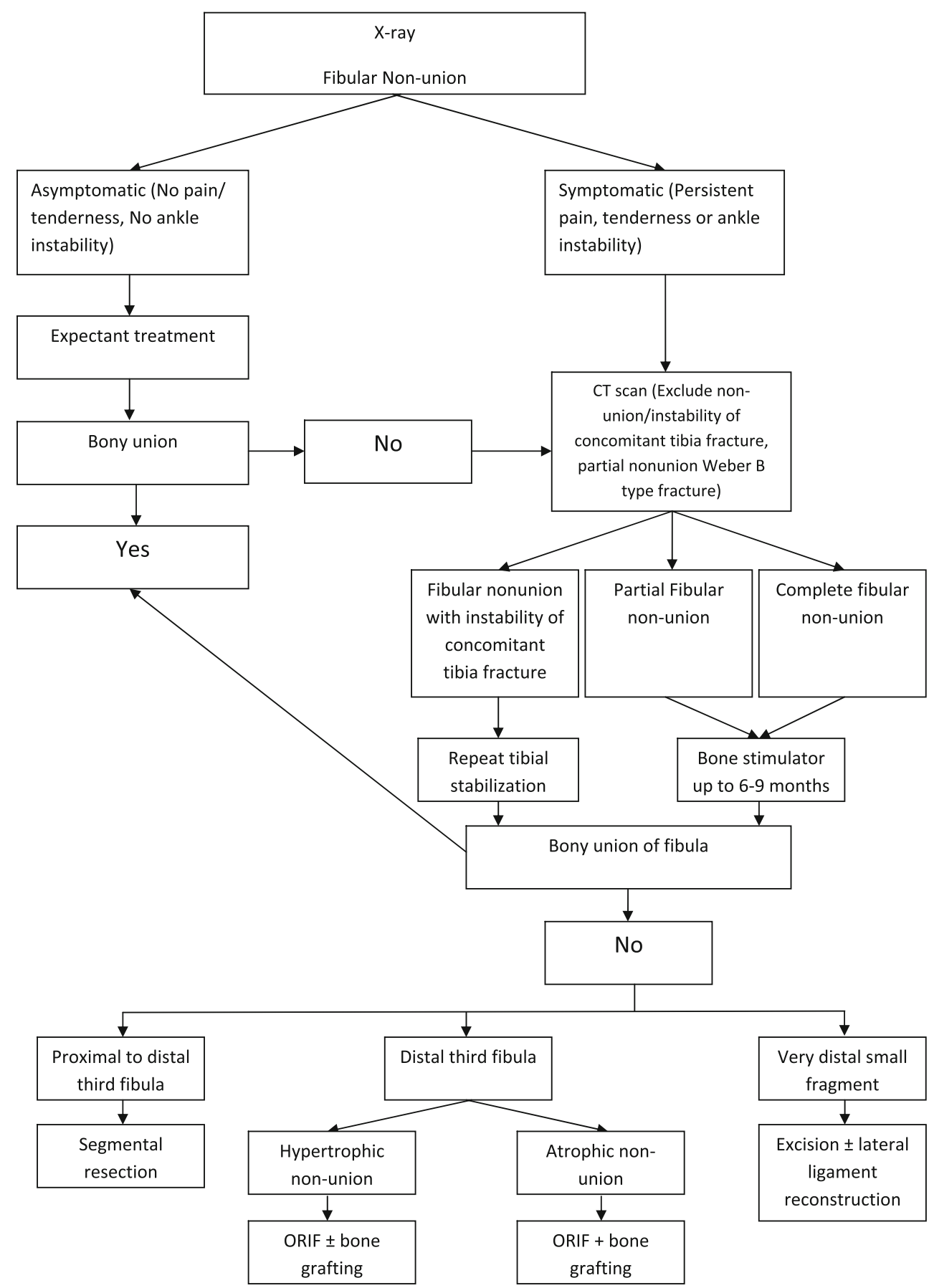


bones, we could not find any such evidence in the current literature for the management of nonunion of the fibula.

Our study was strengthened by including a fairly large number of cases. One limitation was that all the available studies were retrospective case series (EBM - level IV).

In summary, nonunion of the fibula is becoming more common in association with intramedullary nailing of concomitant tibial shaft fractures. We believe the problem of delayed union or frank nonunion of the fibula is not given due importance in orthopaedic literature, and is, hence, underreported. Clinicians should consider the possibility of presence of fibular nonunion in patients with persistent lateral ankle symptoms and warrant $\mathrm{CT}$ scanning to confirm the diagnosis. Although many of these relatively benign cases go on to spontaneous union over a prolonged period, symptomatic nonunion of the fibula should be treated with internal fixation with or without bone grafting depending on type of nonunion and severity of communition at the fracture site.

A treatment algorithm for nonunion of the fibula has been proposed (Fig. 6). The suspicion for nonunion of the fibula should be heightened in lower leg fractures if the patient is symptomatic, and the progression of healing is not as expected. Ideally, prospective, multicentre studies would be performed to provide more rigorous data on the incidence, risk factors, and optimum treatment.

Conflict of interest The authors have no conflict of interest to report.

\section{References}

1. Böstman O, Kyrö A (1991) Delayed union of fibular fractures accompanying fractures of the tibial shaft. J Trauma 31(1):99-102

2. Mendelsohn HA (1965) Nonunion of malleolar fracture of the ankle. Clin Orthop Relat Res 11(233):103-118

3. Sneppen O (1971) Pseudoarthrosis of the lateral malleolus. Acta Orthop Scand 42:187-200

4. Ebraheim NA, Savolaine ER, Skie MC et al (1993) Fibular nonunion in combination with fractures of the tibia. J Orthop 16(11):1229-1232
5. Shen WJ, Shen YS (1993) Fibular nonunion after fixation of the tibia in lower leg fractures. Clin Orthop Rel Res 287:231-232

6. Walsh EF, DiGiovanni D (2004) Fibular nonunion after closed rotational ankle fractures. Foot Ankle Int 25:488-495

7. Sneppen O (1971) Treatment of pseudoarthrosis involving the malleolus: a post-operative follow-up of 34 cases. Acta Orthop Scand 42:201-216

8. Ahmed M, Wimhurst JA, Walton P (2007) Nonunion of Weber B fractures: a case series. Injury 38:861-884

9. Brinker MR, O'Connor DP (2010) Partial fibulectomy for symptomatic fibular nonunion. Foot Ankle Int 31(6):542-546

10. McGonagle L, Ralte P, Kershaw S (2010) Non-union of Weber B distal fibula fractures: a case series. Foot Ankle Surg 16(3):e63-67

11. Panagiotis M (2005) Classification of non-union. Injury 36S:S30-S37

12. Konig M, Gotzen L (1989) Pseudarthroses of the fibula following fractures of the lower leg. Unfallchirurg 92(4):191-194

13. Siliski J, Blitzer C, Healy W, Baumgaertner M, Carr C (1994) Non-union of the fibula after ankle fracture. Orthop Trans 18:22

14. Böhler L (1957) Die Technik der Knochenbruch Behandlung. Maudrich, Wien, pp 2074-2077

15. Kenwright J, Richardson JB, Goodship AE et al (1986) Effect of controlled axial micromovement on healing of tibial fractures. Lancet 2:1185-1187

16. Aaron RK, Ciombor MD, Simon JD (2004) Treatment of nonunion with electric and electromagnetic fields. Clin Orthop Relat Res 419:21-29

17. Guerkov HH, Lonmann CH, Liu Y et al (2001) Pulsed electromagnetic field increase growth factor release by nonunion cell. Clin Orthop Relat Res 384:265-279

18. Crenshaw H (1987) Delayed union and nonunion of fractures. In: Crenshaw AH (ed) Campbell's operative orthopaedics, vol 3. Mosby/Elsevier, Philadelphia, pp 2053-2118

19. Friedlaender GE, Perry CR, Cole JD et al (2001) Osteogenic protein-1 (bone morphogenic protein- 7) in the treatment of tibial nonunion. J Bone Joint Surg Am 83A(Suppl 1):51-58

20. Guo F (1981) Observations of the blood supply to the fibula. Arch Orthop Traumat Surg 98:147-151

21. Hernigou P, Poignard A, Beaujean (2005) Percutaneous autologous bone marrow grafting for nonunion. J Bone Joint Surg Am $87: 1430-1437$

22. McKee M (2000) Aseptic non-union In: Ruedi TP, Murphy WM (eds) AO principles of fracture management, Stuttgart, New York, 748-762

23. Rodriguez-Merchan CE, Forriol F (2004) Nonunion: general principles and experimental data. Clin Orthop Relat Res 419:4-12

24. Uchiyama E, Suzuki D, Kura H, Yamashita T, Murakami G (2006) Distal fibula length needed for ankle stability. Foot Ankle Int 27:185-189

25. Weber BG, Cech O (1976) Pseudoarthrosis. Grune and Stratton, New York 